\section{Vaginal bacteria modify HIV tenofovir microbicide efficacy in African women}

\author{
Nichole R. Klatt, ${ }^{1 *} †$ Ryan Cheu, ${ }^{1} \ddagger$ Kenzie Birse, ${ }^{2,3} \ddagger$ Alexander S. Zevin, ${ }^{1} \ddagger$ \\ Michelle Perner, ${ }^{2,3} \ddagger$ Laura Noël-Romas, ${ }^{2,3} \ddagger$ Anneke Grobler, ${ }^{4}$ Garrett Westmacott, ${ }^{5}$ \\ Irene Y. Xie, ${ }^{2,3}$ Jennifer Butler, ${ }^{2,3}$ Leila Mansoor, ${ }^{4}$ Lyle R. McKinnon, ${ }^{3,4}$ \\ Jo-Ann S. Passmore, ${ }^{6,4}$ Quarraisha Abdool Karim, ${ }^{4,7}$ \\ Salim S. Abdool Karim, ${ }^{4,7}$ Adam D. Burgener ${ }^{2,3,8 *} \dagger$
}

Antiretroviral-based strategies for HIV prevention have shown inconsistent results in women. We investigated whether vaginal microbiota modulated tenofovir gel microbicide efficacy in the CAPRISA (Centre for the AIDS Program of Research in South Africa) 004 trial. Two major vaginal bacterial community types-one dominated by Lactobacillus $(59.2 \%)$ and the other where Gardnerella vaginalis predominated with other anaerobic bacteria $(40.8 \%)$-were identified in 688 women profiled. Tenofovir reduced HIV incidence by $61 \%(P=0.013)$ in Lactobacillus-dominant women but only $18 \%(P=0.644)$ in women with non-Lactobacillus bacteria, a threefold difference in efficacy. Detectible mucosal tenofovir was lower in non-Lactobacillus women, negatively correlating with $G$. vaginalis and other anaerobic bacteria, which depleted tenofovir by metabolism more rapidly than target cells convert to pharmacologically active drug. This study provides evidence linking vaginal bacteria to microbicide efficacy through tenofovir depletion via bacterial metabolism.

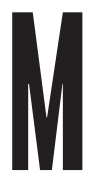
ore than 1 million women are infected with HIV annually, and the majority of these new infections occur in young women in sub-Saharan Africa, with South Africa having among the highest incidence rates $(1,2)$. Antiretroviral-based clinical trials in men who have sex with men have consistently demonstrated effectiveness in preventing HIV infection (3-5); however, studies in women have produced widely varying results. In clinical trials of women, the efficacy of antiretroviral drugs to prevent HIV infection ranged from $-49 \%$ [VOICE (Vaginal and Oral Interventions to Control the Epidemic) study] to $75 \%$ [TDF2 (Tenofovir Disoproxil Fumarate Two) study] for daily oral tenofovir or tenofoviremtricitabine and from 0\% [FACTS (Follow-on

\footnotetext{
${ }^{1}$ Department of Pharmaceutics, Washington National Primate Research Center, University of Washington, Seattle, WA, USA. ${ }^{2}$ National HIV and Retrovirology Labs, J.C. Wilt Infectious Diseases Research Centre, Public Health Agency of Canada, Winnipeg, Manitoba, Canada. ${ }^{3}$ Department of Medical Microbiology and Infectious Diseases, University of Manitoba, Winnipeg, Manitoba, Canada. ${ }^{4}$ Centre for the AIDS Program of Research in South Africa (CAPRISA), University of KwaZuluNatal, Durban, South Africa. ${ }^{5}$ Mass Spectrometry and Proteomics Core Facility, National Microbiology Laboratory, Public Health Agency of Canada, Winnipeg, Manitoba, Canada. ${ }^{6}$ Institute of Infectious Diseases and Molecular Medicine (IDM) University of Cape Town and National Health Laboratory Service, Cape Town, South Africa. ${ }^{7}$ Department of Epidemiology, Mailman School of Public Health, Columbia University, NY, USA. ${ }^{8}$ Unit of Infectious Diseases, Department of Medicine Solna, Center for Molecular. Medicine, Karolinska Institute, Karolinska University Hospital, Stockholm, Sweden.

*Corresponding author. Email: adam.burgener@umanitoba.ca (A.D.B.); klattnr@uw.edu (N.R.K.)

†These authors contributed equally to this work. $\ddagger$ These authors contributed equally to this work.
}

African Consortium for Tenofovir Studies) 001] to $39 \%$ [CAPRISA (Centre for the AIDS Program of Research in South Africa) 004] for daily or coital vaginally-applied tenofovir gel (fig. S1). Variability in the levels of adherence (6) has been shown to be a major contributing factor for the diverse trial outcomes in women. However, little is known about what biological factors may also contribute to the variability in these results and why higher adherence is required for antiretroviralbased prevention efficacy in women (7).

The vaginal compartment contains many microbial species critical for the health of the vaginal mucosa, and dysbiosis of vaginal bacteria, clinically known as bacterial vaginosis (BV), can result in negative reproductive health outcomes $(8,9)$. The recent advent of advanced molecular tools has redefined our understanding of vaginal bacteria communities $(10,11)$, where the most frequently observed community state types (CSTs) have been described (11-14). Although substantial heterogeneity exists, a key commonality is that CSTs fall into two clear groups: (i) Lactobacillus-dominant, where one or more species of Lactobacillus make up $>90 \%$ of the total copy number or sequencing reads (L. iners, L. crispatus, L. jensenii, and L. gasseri), and (ii) non-Lactobacillus-dominant, with Lactobacillus making up $<30 \%$ of the total copy number or sequencing reads. The non-Lactobacillus-dominant group typically contains a high abundance of Gardnerella vaginalis alone or codominant with other facultative and obligate anaerobic bacteria, including Prevotella ssp., Mobiluncus ssp., and/or several Clostridia species. BV occurs after a shift from Lactobacillus dominance to these more diverse communities $(11,12)$, is frequently asymptomatic, and can often go undetected using traditional Amsel's criteria (15) and/or the Nugent's score used to diagnose BV (16).

Bacterial vaginosis is associated with poor reproductive health outcomes and increased HIV infection risk in women (17), by as much as $60 \%$ in some meta-analyses of women with BV (18). BV likely increases HIV risk through multiple mechanisms, including increased inflammation and target cells, as well as vaginal epithelial barrier disruption and wound-healing impairment; however, the mechanisms are not entirely understood $(13,19,20)$. Given that women from subSaharan Africa have high prevalence rates of BV (21) and that HIV prevention strategies are being targeted for women in these areas, we investigated whether vaginal microbial communities may affect the efficacy of antiretroviral-based prevention technologies, especially those that are topically applied to the vaginal surface.

Characterization of the vaginal microbiome using unbiased metagenomic, metatranscriptomic, and metaproteomic approaches represents a potential paradigm shift in understanding hostmicrobial interactions at the mucosal surface in vivo. We recently used metaproteomics to gain insight into host-bacterial interactions in vaginal microbial dysbiosis (20). This method simultaneously collects unbiased information on microbial and host proteomes, thus providing systems-level information on microbial communities and mucosal surfaces not available with other techniques. In this work, we used a metaproteomic approach to assess whether vaginal bacteria modulate the efficacy of the topical microbicide tenofovir in preventing HIV infection, and we also used in vitro systems to determine mechanisms of microbiome influence on tenofovir.

\section{Vaginal microbial diversity in women using tenofovir or placebo microbicide gels}

Samples from 688 HIV-negative women that were assigned to either the tenofovir or the placebo-gel arm were analyzed by protein mass spectrometry, as outlined in the materials and methods (fig. S2). Proteomic analysis identified 3334 distinct bacterial proteins from 188 different species in the cervicovaginal lavage (CVL) samples of 688 women. Two major vaginal bacterial community groups were identified: one in which Lactobacillus was the predominant genus (group I) ( $n=423$ women, $61.5 \%$ ) and the other dominated by nonLactobacillus microbiota (group II) ( $n=265$ women, 38.5\%) (Fig. 1). Approximately $11 \%$ of individuals had no single dominant species (defined as $>50 \%$ community composition), and the majority of these individuals fell into group II.

In comparing the mass spectrometry proteomic approach with $16 S$ ribosomal RNA (rRNA) sequencing, we found concordance with respect to classifying women into groups I and II (91.5\% agreement) and measurements of bacterial abundance, including major taxa Lactobacillus, G. vaginalis, Prevotella, and others $(P<0.001)$ (fig. S3). A limitation of this study was that clinical BV data was not collected during the CAPRISA 004 trial, 
A

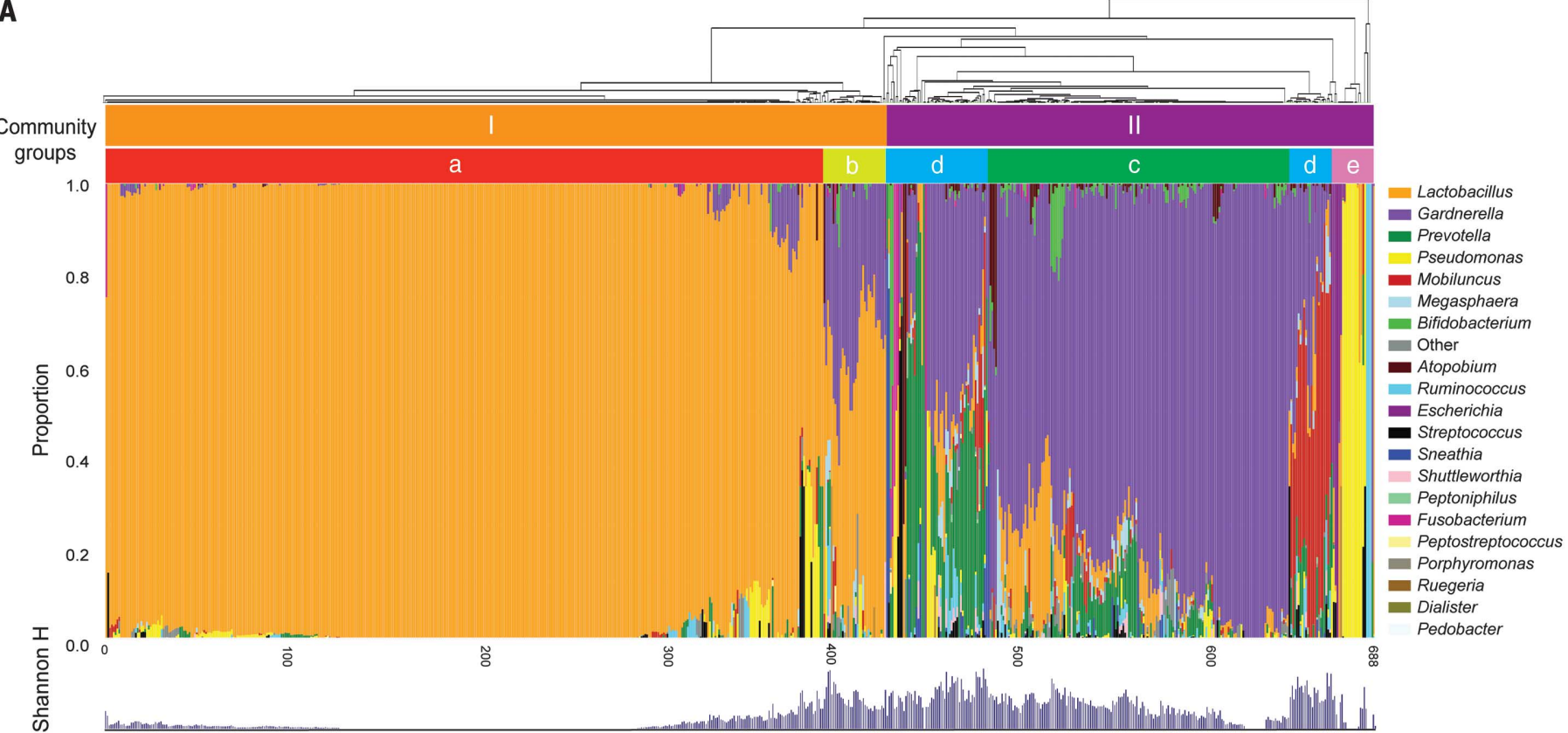

B
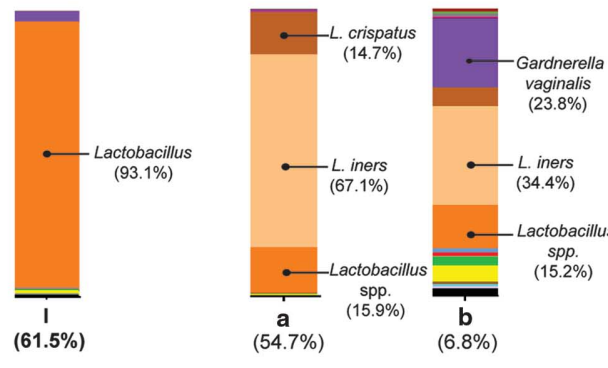

C
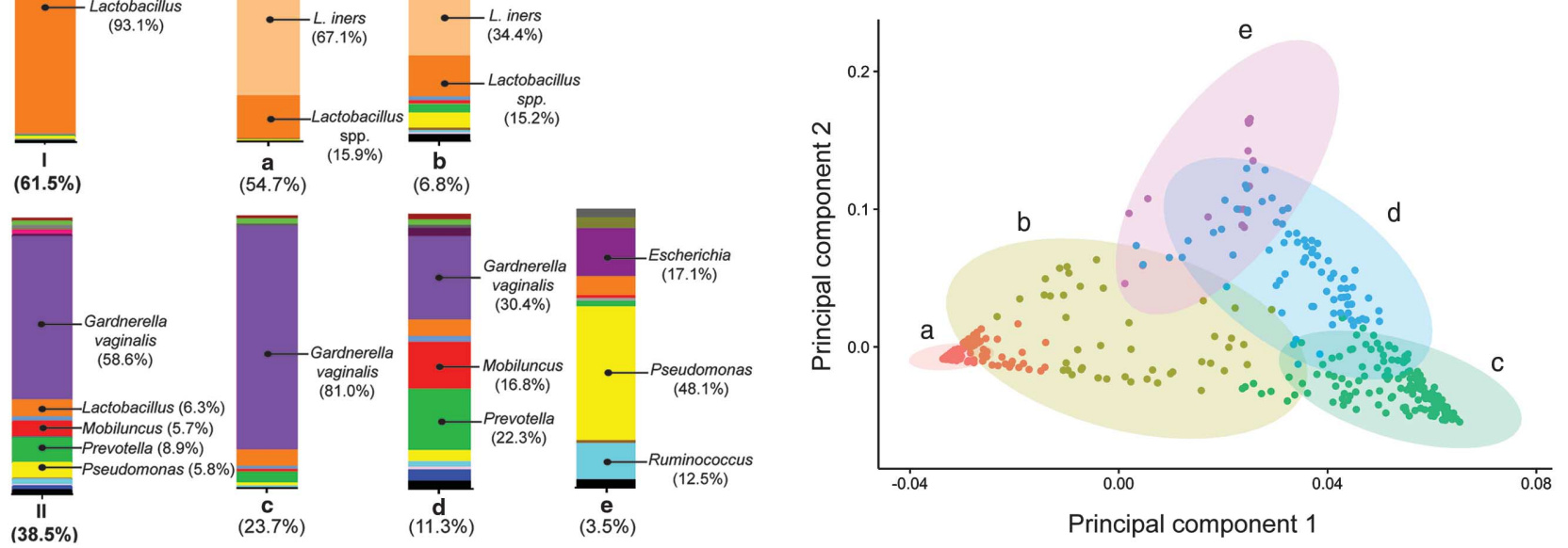

Fig. 1. Bacterial profiling by mass spectrometry using cervicovaginal lavage samples from 688 women from the CAPRISA 004 trial. (A) Overall bacterial diversity plot of the major genera of all women profiled. (B) Average bacterial community group structure for each of the two major profiles: group I (subgroups $\mathrm{a}$ and b) and group II (subgroups c, d, and e). (C) Principal components analysis of 688 women using bacterial proportion data showing the five subgroups.

thus precluding comparisons to Nugent score or other BV criteria.

Group I showed the lowest diversity [Shannon diversity index (H-index) median: 0.05], with the majority (70.5\%) having L. iners as the dominant species, followed by L. crispatus (15.1\%) and other lactobacilli, such as $L$. jensenii and $L$. gasseri (3.0\%). In Fig. 1B, the first subgroup (a) was homogeneous, with clear dominance of Lactobacillus (54.7\%) with very low diversity (H-index median: 0.035), whereas a minority of these individuals (subgroup b, 6.8\%) showed an intermediate amount of diversity (H-index median: 0.87 ) where small amounts of G. vaginalis, Pseudomonas, and other bacteria were detected. A subanalysis of group Ia at the Lactobacillus species level clearly illustrates L. iners as predominant (fig. S4).

Women in group II had higher overall bacterial diversity (H-index: 0.78) with several distinct subgroups. The largest subgroup (c) was dominated by G. vaginalis ( $n=163,23.7 \%$, H-index: 0.66 ) and contained multiple taxa, including Prevotella and minor amounts of Lactobacillus and Mobiluncus (Fig. 1B). The second-largest subgroup (d) was the most diverse and had no one clearly dominant taxa ( $n=78,11.3 \%$, H-index: 1.15); G. vaginalis, Prevotella, and Mobiluncus were predominant. Finally, the smallest subgroup (e) was low in diver- sity, containing either Pseudomonas or Escherichia ( $n=24,3.5 \%$, H-index: 0.14) (Fig. 1B). Grouping subgroups a and b, which showed relative homogeneity in a single group (group I), with the remaining three subgroups (c, d, and e), which had more variability and diversity into a single group (group II), was supported by principal components analysis (Fig. 1C). Overall, the majority $(96.2 \%, n=$ 407) of women in group I had $>50 \%$ Lactobacillus by abundance.

For downstream comparisons of topical tenofovir efficacy, we chose a Lactobacillus-dominant $(>50 \%)(\mathrm{LD})$ or non-Lactobacillus-dominant $(\leq 50 \%)$ (non-LD) classification, as $>50 \%$ Lactobacillus at 


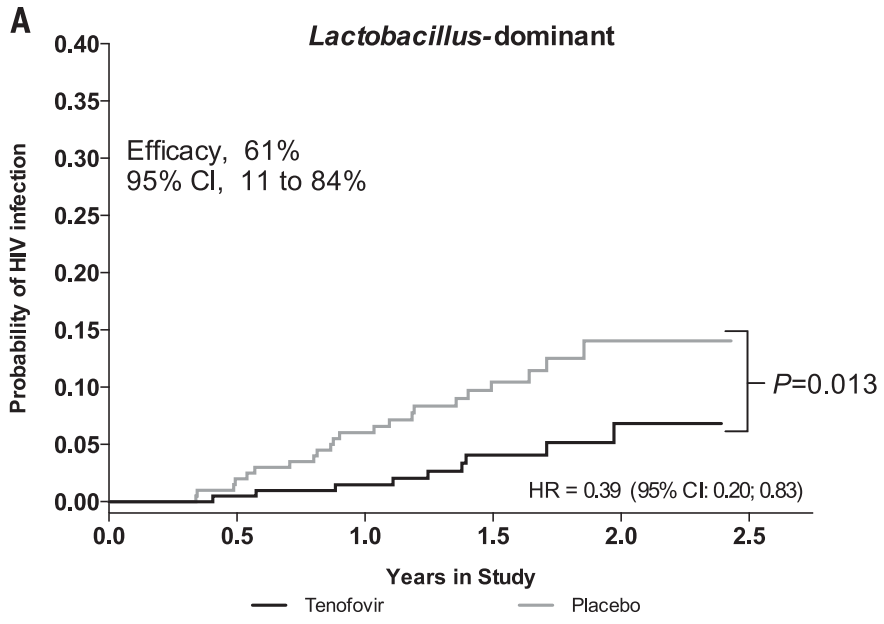

Lactobacillus-dominant women at risk (Cumulative number of infections)

Tenofovir $205(0) \quad 204(1) \quad 183(3) \quad 129(7) \quad 46(9) \quad 0(9)$

B

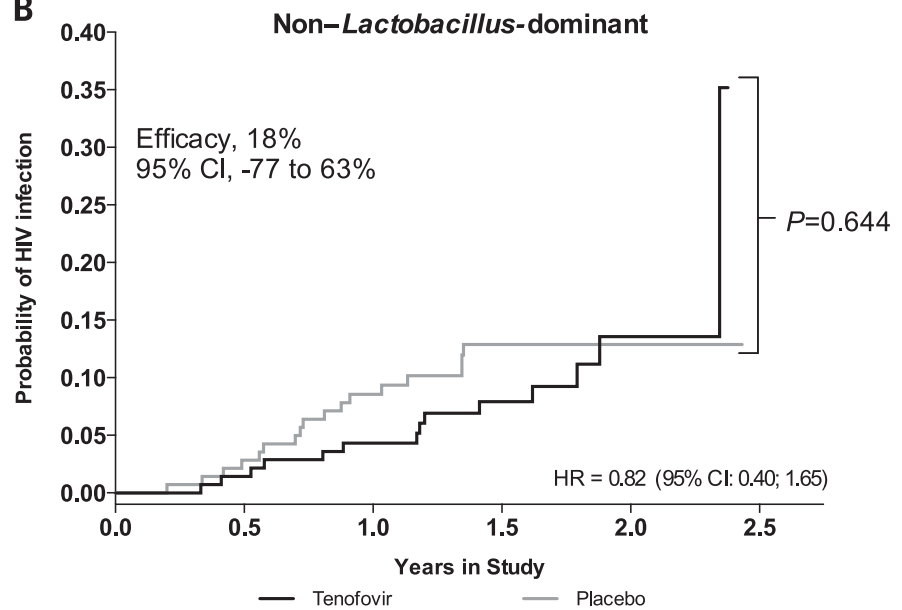

Non-Lactobacillus-dominant women at risk (Cumulative number of infections)

$\begin{array}{lcccccc}\text { Tenofovir } & 140(0) & 137(2) & 123(6) & 87(10) & 32(13) & 0(14) \\ \text { Placebo } & 141(0) & 137(4) & 116(12) & 84(17) & 28(17) & 0(17)\end{array}$

Fig. 2. Cumulative HIV infection probability by treatment assignment in women with vaginal Lactobacillus dominance and non-Lactobacillus bacterial dominance. Data for (A) Lactobacillus-dominant (Lactobacillus > 50\%) $(n=407)$ and (B) non-Lactobacillus-dominant (Lactobacillus $\leq 50 \%)$ $(n=281)$ women. The tables below each panel show the cumulative number of HIV infections in each study arm, corresponding HIV incidence rates, and efficacy of tenofovir gel to prevent HIV acquisition for each additional 6 months of follow-up. The protective efficacy of tenofovir gel was more than threefold higher in women with Lactobacillus dominance (A) compared with non-Lactobacillus dominance (B). HR, hazard ratio.

the proteome level corresponds with a practical clinical marker of vaginal $\mathrm{pH}$ below 4.5 [interquartile range $\mathrm{pH}$ value of 4.0 to $4.5(20)]$ and accurately identified $96.2 \%$ of group I individuals. The use of LD and non-LD definitions did not appreciably change any outcomes reported on the basis of group I versus group II comparisons.

Both LD and non-LD women had similar baseline clinical, behavioral, and demographic characteristics, as well as sexual behavior and gel adherence during the trial (table S1). The presence of a sexually transmitted infection (STI)-which included Chlamydia trachomatis, Trichomonas vaginalis, Neisseria gonorrhea, Mycoplasma genitalium, Treponema pallidum, and herpes simplex virus 2 (HSV-2)-was comparable between groups. Although not statistically significant, women within the non-LD group were marginally younger (mean age 23.6 versus 24.1 years, $P=0.092$ ). Within each of the groups of $\mathrm{LD}$ and non-LD women, the number of individuals assigned to tenofovir gel and placebo gel was similar (table S2).

\section{Vaginal microbial profiles and tenofovir gel efficacy}

In the LD group, the HIV incidence rate was $61 \%$ lower [95\% confidence interval (CI): 0.16 to 0.89 ] in women assigned to tenofovir gel compared with those assigned to the placebo gel [ 2.7 versus 6.9 per 100 women-years (where 1 woman-year is defined as 1 year of study observation of one woman); incidence rate ratio $($ IRR $)=0.39 ; P=0.013$ ] (Fig. 2A). In contrast, in non-LD women, the HIV incidence rate was only $18 \%$ lower ( $95 \%$ CI: 0.37 to 1.77) in those assigned to tenofovir gel compared with those assigned to placebo gel (6.4 versus 7.8 per 100 women-years; IRR $=0.82 ; P=0.644$ ) (Fig. $2 B)$. Adjusting for STIs (including HSV-2 infec- tion), antibiotic usage, depot medroxyprogesterone acetate (DMPA) usage, and sexual behaviors (frequency of sex, number of partners, and condom usage) did not affect these findings (table S3).

Lactobacillus, particularly L. crispatus, has been associated with reduced HIV infection. Although the HIV incidence rate of 4.8 per 100 womenyears in LD women was $32 \%$ lower (95\% CI: 0.4 to 1.12 ) than the 7.1 per 100 women-years in nonLD women, this was not statistically significant $(P=0.127$ ) (fig. S5). Comparing the subgroup of all $63 \mathrm{~L}$. crispatus-dominant ( $>50 \%$ abundance) to non-LD women yielded a $57 \%$ lower HIV incidence (95\% CI: 0.13 to 1.41 ) that was not statistically significant (3.1 versus 7.1 per 100 women-years; $P=0.167$ ) (fig. S6). Comparison of $L$. crispatus-dominant women to all others as a single group (L. iners-dominant and non-LD women) produced similar findings $(50 \%$ lower HIV incidence; 3.1 versus 6.0 per 100 womenyears; $95 \%$ CI: 0.16 to $1.60, P=0.237$ ). Similarly, within the placebo group, the $31 \mathrm{~L}$. crispatusdominant women compared with non-LD women showed a 48\% lower HIV incidence (95\% CI: 0.12 to 1.27 ) that was not statistically significant (4.17 versus 7.8 per 100 women-years; $P=0.387$ ) (fig. S7). This is likely attributed to insufficient power due to the low numbers of women with L. crispatus dominance. However, because the proportion of women with $L$. crispatus dominance was similar in women assigned to tenofovir gel and placebo gel (51\% versus $49 \%$ ), the HIV incidence differences between these two groups of women are not due to $L$. crispatus. In addition, considering just $L$. iners-dominant women, the efficacy of tenofovir was maintained, where the HIV incidence rate was $67 \%$ lower (95\% CI: 0.13 to 0.83 ) in those assigned to tenofovir gel compared with those assigned to placebo gel ( 2.5 versus 7.7 per 100 women-years in the tenofovir and placebo arms, respectively; $P=0.0118$ ) (fig. S8).

Microbicide gel adherence and tenofovir efficacy in LD and non-LD women

Gel adherence, as assessed by monthly empty applicator returns (22), was similar in both groups: $60.0 \%$ [244 of $407(244 / 407)$ ] of LD women compared to $61.4 \%(172 / 280)$ of non-LD women had $>50 \%$ gel adherence (where $>50 \%$ of sex acts were covered by two applications of the gel, as recommended in the trial) (Table 1). Stratifying $\mathrm{LD}$ and non-LD women separately on adherence demonstrates that gel adherence $>50 \%$ was associated with higher efficacy in preventing HIV in $\mathrm{LD}$ women than in non-LD women (Table 1). The efficacy of tenofovir gel in preventing HIV infection in the subgroup of women with $>50 \%$ adherence was $78 \%$ (95\% CI: $29 \%, 95 \% ; P=0.003$ ) in the LD group but only $26 \%$ (95\% CI: $-98 \%$, $73 \% ; P=0.558)$ in the non-LD group.

\section{Vaginal tenofovir concentrations are lower in non-LD versus LD women}

Tenofovir concentrations $(n=270)$ were assessed in a random sample of CVLs from HIV-negative women and from the first postinfection visit CVL from HIV seroconvertors. Although gel adherence was not different between the $\mathrm{LD}$ and non- $\mathrm{LD}$ groups and time since the last gel application was similar $(P=0.558)$ (table $\mathrm{S} 1)$, tenofovir was only detectable in CVL samples in $29.8 \%(34 / 114)$ of non-LD women compared with $46.2 \%$ (72/156) of LD women $(P=0.008)$. Genital tenofovir concentrations were also significantly lower in non$\mathrm{LD}$ (upper quartile $=24.3 \mathrm{ng} / \mathrm{ml}$ ) compared with LD women (upper quartile $=8020 \mathrm{ng} / \mathrm{ml}$ ) 
A

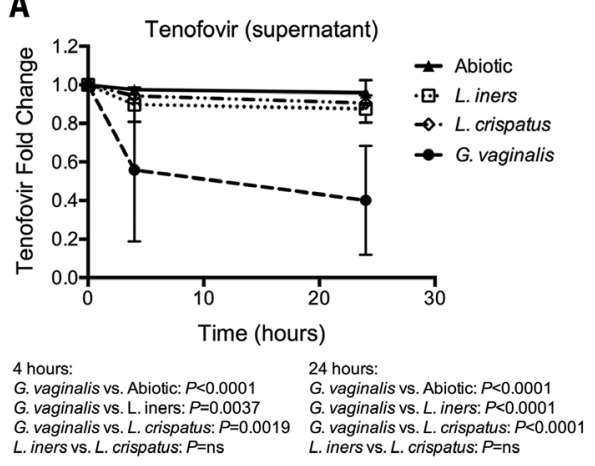

D

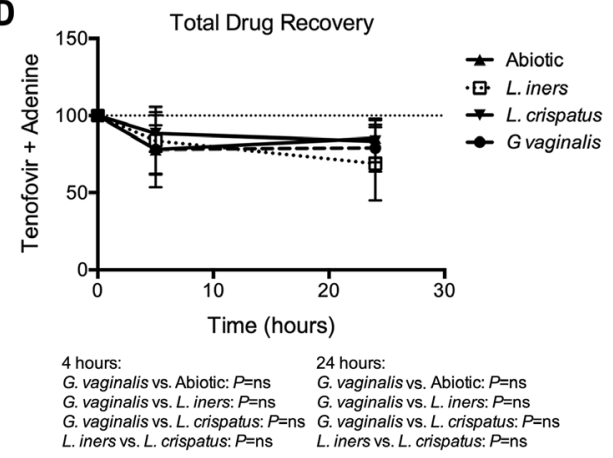

Fig. 3. Metabolism of tenofovir by G. vaginalis and BV-associated

bacteria. (A) Tenofovir fold change in supernatants after $1 \mathrm{mg} / \mathrm{ml}$ tenofovir was added to $G$. vaginalis, $L$. iners, and $L$. crispatus cultures or abiotic controls in NYCIII media. Tenofovir levels were measured by mass spectrometry at 0, 4 and 24 hours. Data show average \pm SEM (error bars) of 18 replicate experiments for $L$. iners and $G$. vaginalis cultures compared with 15 replicates of abiotic controls. (B) Total intracellular tenofovir detected in cell pellets from cultures. (C) Predicted tenofovir metabolite

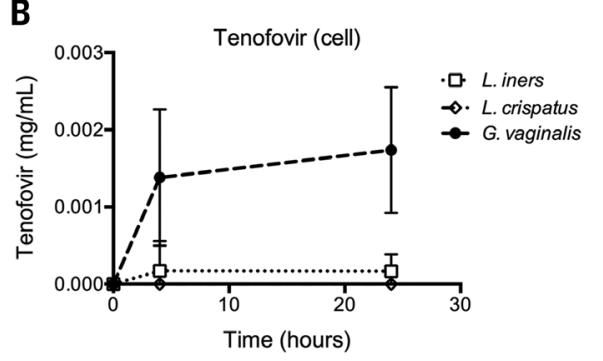

C

Tenofovir

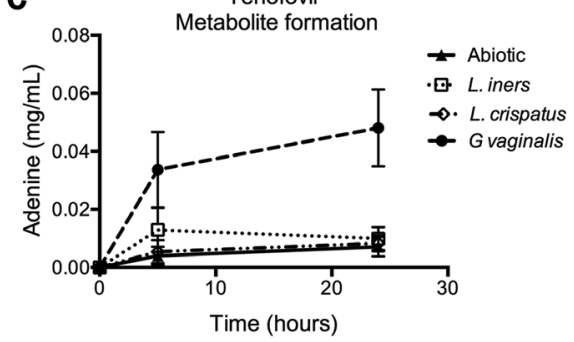

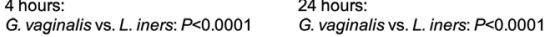
$\begin{array}{ll}\text { G. vaginalis vs. L. crispatus: } P<0.0001 & G \text {. vaginalis vs. L. crispatus: } P<0.0001\end{array}$ L. iners vs. L. crispatus: $P=n s$

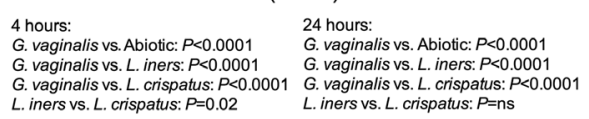

$\mathbf{E}$

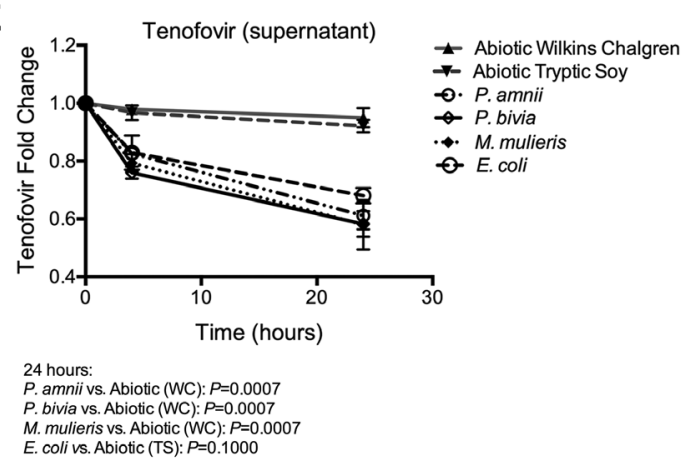

$(P=0.0077)$. A subanalysis showed that genital tenofovir concentrations negatively correlated with $G$. vaginalis protein abundance (correlation coefficient $r=-0.19, P=0.0014)$ and other anaerobic bacteria (Prevotella, $r=-0.14, P=0.023$ ), suggesting a relationship between $\mathrm{BV}$-associated bacteria and tenofovir levels.

\section{Metabolism by G. vaginalis and BV-associated bacteria leads to tenofovir depletion}

Given the decreased levels of mucosal tenofovir in non-LD-dominant women with $G$. vaginalis and other BV-associated bacteria versus LD women, we aimed to determine whether interactions between microbes and tenofovir may underlie altered drug levels. We used an in vitro culture system to assess potential biodegradation of tenofovir by the major bacterial species present in this cohort. We found that tenofovir concentrations in culture with G. vaginalis decreased rapidly by $50.6 \%$ compared with marginal changes in either $L$. iners $(P=0.0037)$, L c crispatus $(P=$ 0.0019 ), or abiotic (same NYCIII media without bacteria) control $(P<0.0001)$ at 4 hours (Fig. 3A). The differential decline continued; by 24 hours, tenofovir concentrations in the culture medium had dropped $67.4 \%$ with $G$. vaginalis but only
14.0\% with L. iners $(P<0.0001)$ and $9.4 \%$ with L. crispatus $(P<0.0001)$ (Fig. $3 \mathrm{~A})$. Of interest, G. vaginalis used here was a subtype $\mathrm{C}$ strain (ATCC type strain 14018), but repeating these methods with G. vaginalis with Kenyan clinical isolates from three different subtypes demonstrated that all subtypes of $G$. vaginalis metabolized tenofovir $(P<0.005)$ (fig. S9). Concomitantly to tenofovir loss, intracellular tenofovir concentrations rose sharply in $G$. vaginalis but not in L. iners or L. crispatus cultures $(P<0.0001)$ (Fig. 3B). Predicted metabolites at mass/charge ratios $(\mathrm{m} / \mathrm{z})$ of $136.06,206.10,159.07$, and 59.05 showed a sharp increase at $136.06 \mathrm{~m} / \mathrm{z}$ in $\mathrm{G}$. vaginalis cultures, indicating adenine production via cleavage of oxy-methylphosphonic acid (Fig. 3C), the side-chain component of tenofovir $(P<0.0001$ compared with L. crispatus, L. iners, and abiotic). Residual tenofovir plus the intracellular metabolite adenine made up $>80 \%$ of recovered products, indicating that adenine is the major metabolite of tenofovir metabolism by $G$. vaginalis (Fig. 3D). Finally, to determine whether other major bacterial species in non-LD women were capable of metabolizing tenofovir, we tested the ability of P. amnii, P. bivia, Mobiluncus mulieris, and Escherichia coli to deplete tenofovir. We found that both Prevotella species and M. mulieris sig- nificantly depleted tenofovir compared with abiotic (Wilkins-Chalgren media) controls $(P=0.0007$ for all at 24 hours), and $E$. coli trended toward depletion ( $P=0.100$ as compared to abiotic tryptic soy media), though not to the same extent or as rapidly as G. vaginalis (Fig. 3E).

\section{Metabolism by vaginal bacteria affects uptake and conversion of tenofovir to active drug in target cells}

We next assessed whether tenofovir metabolism by bacteria affects the kinetics or ability of target cells to uptake tenofovir and convert to pharmacologically active tenofovir diphosphate. We performed cocultures of Jurkat cells (HIV targets) in the presence of tenofovir, with G. vaginalis, L. iners, L. crispatus, or abiotic controls (both tenofovir alone or tenofovir plus Jurkat cells), to assess overall tenofovir depletion and uptake in culture. In these Jurkat cell cultures, we found that tenofovir is most rapidly depleted in the presence of $G$. vaginalis, relative to Jurkat cells alone $(P=$ $0.0001)$ or those with $L$. iners $(P=0.0022)$ or L. crispatus $(P=0.0238)$ (Fig. $4 \mathrm{~A})$. To assess microbial tenofovir metabolism, we measured adenine levels in the cell pellets and found that adenine was created only in cultures with $G$. vaginalis $(P=0.0022$ relative to all conditions) (Fig. 4B). A 
Table 1. Effect of adherence on the HIV prevention efficacy of $1 \%$ tenofovir gel in women participating in the CAPRISA 004 trial, stratified by Lactobacillus dominance in the female genital tract. One woman did not produce adherence data. $n$, number of women.

No. of HIV infections/women-years

\begin{tabular}{|c|c|c|c|c|c|c|c|c|}
\hline Gel adherence & Tenofovir & Placebo & $n$ & Tenofovir & Placebo & Incidence rate ratio & Efficacy & $P$ value (log-rank) \\
\hline \multicolumn{9}{|c|}{ All participants } \\
\hline Lactobacillus-dominant & $9 / 331$ & $22 / 318$ & 407 & $2.7(1.2 ; 5.2)$ & $6.9(4.3 ; 10.5)$ & $0.39(0.16 ; 0.89)$ & $61 \%$ & 0.013 \\
\hline Non-Lactobacillus-dominant & $14 / 219$ & $17 / 218$ & 281 & $6.4(3.5 ; 10.7)$ & $7.8(4.5 ; 12.5)$ & $0.82(0.37 ; 1.77)$ & $18 \%$ & 0.644 \\
\hline Greater than $50 \%$ adherence & $13 / 349$ & $26 / 304$ & 416 & $3.7(2.0 ; 6.4)$ & $8.6(5.6 ; 12.5)$ & $0.44(0.21 ; 0.88)$ & $56 \%$ & 0.013 \\
\hline Less than $50 \%$ adherence & $10 / 200$ & $13 / 232$ & 271 & $5.0(2.4 ; 9.2)$ & $5.6(3.0 ; 9.6)$ & $0.89(0.35 ; 2.21)$ & $11 \%$ & 0.771 \\
\hline \multicolumn{9}{|c|}{ Lactobacillus-dominant } \\
\hline Greater than $50 \%$ adherence & $4 / 209$ & $15 / 176$ & 244 & $1.9(0.5 ; 4.9)$ & $8.5(4.8 ; 14.1)$ & $0.22(0.05 ; 0.71)$ & $78 \%$ & 0.003 \\
\hline Less than $50 \%$ adherence & $5 / 122$ & $7 / 142$ & 163 & $4.1(1.3 ; 9.6)$ & $4.9(2.0 ; 10.2)$ & $0.83(0.21 ; 3.05)$ & $17 \%$ & 0.735 \\
\hline \multicolumn{9}{|c|}{ Non-Lactobacillus-dominant } \\
\hline Greater than $50 \%$ adherence & $9 / 141$ & $11 / 128$ & 172 & $6.4(2.9 ; 12.2)$ & $8.6(4.3 ; 15.4)$ & $0.74(0.27 ; 1.98)$ & $26 \%$ & 0.558 \\
\hline Less than $50 \%$ adherence & $5 / 78$ & $6 / 90$ & 108 & $6.4(2.1 ; 15.0)$ & $6.7(2.4 ; 14.5)$ & $0.96(0.23 ; 3.79)$ & $4 \%$ & 0.935 \\
\hline
\end{tabular}

critical component is whether G. vaginalis can metabolize tenofovir more rapidly than target cells can convert tenofovir to pharmacologically active tenofovir diphosphate. To assess this, we measured tenofovir diphosphate in cell pellets and found that although tenofovir diphosphate was made at equal levels in Jurkat cells alone and the presence of Lactobacillus spp., it was significantly decreased in the presence of $G$. vaginalis ( $P=0.0002, P=0.0022$, and $P=0.0238$ relative to tenofovir + Jurkat alone, tenofovir + Jurkat + L. iners, or tenofovir + Jurkat + L. crispatus, respectively) (Fig. 4C). Total drug recovery measurements (tenofovir + tenofovir diphosphate + adenine) demonstrated that all components of tenofovir were fully recovered (Fig. 4D). These data demonstrate that G. vaginalis is capable of decreasing pharmacologically active tenofovir diphosphate by metabolizing tenofovir before drug uptake by target cells.

\section{Implications for antiretroviral-based HIV prevention}

The efficacy of tenofovir-containing topical microbicide to prevent HIV infection varied more than threefold depending on vaginal bacterial profiles; tenofovir gel reduced HIV incidence by $61 \%$ in LD women but only by $18 \%$ in non-LD women. These efficacy differences between LD and non-LD women were consistently present in the most geladherent women ( $78 \%$ versus $26 \%$ ), as well as in the least adherent women (17\% versus $4 \%$ ). In vitro studies demonstrated that metabolism of tenofovir occurred by G. vaginalis, P. bivia, P. amnii, and $M$. mulieris, and slightly by $E$. coli, but not by L. iners or L. crispatus, indicating a putative mechanism for the observed differences in topical microbicide efficacy. The modifying effect of Lactobacillus dominance on tenofovir gel efficacy underscores the importance of both high adherence and $L D$ vaginal bacterial communities for women to benefit maximally from topical microbicides for HIV prevention.

In searching for an underlying cause for the discordance between applicator adherence and detectable vaginal tenofovir concentrations between the LD and non-LD groups, we found that non-Lactobacillus-dominant bacteria associated with BV rapidly metabolized tenofovir, thereby likely reducing extracellular drug availability. The rapid loss of tenofovir by $G$. vaginalis and other bacteria may also affect gel adherence estimates based on vaginal drug levels in non-LD women, particularly considering that Gardnerella metabolizes tenofovir more rapidly than target cells uptake and convert the drug to active form. Thus, although adherence is important for efficacy $(23,24)$, the lack of Lactobacillus dominance may also be a contributing factor. These data indicate that women with BV may have to be more rigorous in their adherence to tenofovir gel administration to be protected against HIV infection, due to the rapid metabolism of tenofovir by dysbiotic bacteria. Because of the potential interplay between vaginal bacteria and adherence in topical microbicide efficacy, a useful next step could be to evaluate the proportion of non-LD women in other topical microbicide trials of tenofovir conducted in Africa such as VOICE and FACTS $(23,24)$. These findings may have broader implications for other topical antiretroviral delivery strategies, such as vaginal rings, for tenofovirbased HIV prevention.

The prevalence of Lactobacillus dominance in women in CAPRISA 004 (59\%) was comparable to that observed in self-described black women from a North American study (58\%) (12) but considerably lower than that reported in Caucasian women (90\%). However, the extent of Lactobacillus dominance may differ even within the same country or region. The Lactobacillus species and overall CSTs in our study participants were similar to those described in a previous South African study $(13,25)$ but somewhat different from those reported recently by the FRESH (Females Rising through Education, Support, and Health) cohort in KwaZulu-Natal in South Africa (13). The reasons for these differences in the prevalence of Lactobacillus dominance are not known. Some notable differences were that the CAPRISA 004 women were slightly older (24 versus 21 years) and had higher hormonal contraceptive usage (97\% versus $54 \%$ on DMPA, norethisterone oenanthate, or combined oral contraceptive pill), both of which have previously been associated with higher Lactobacillus $(26,27)$.

Although Lactobacillus has been associated with lower HIV incidence in previous observational studies, we did not find a strong relationship between LD status and HIV protection in the placebo arm. This may be due to the predominance of $L$. iners in the women in our study, rather than $L$. crispatus, which has been associated with lower HIV incidence $(19,28)$ [whereas L. iners has been associated with increased HIV risk (29)], or simply being underpowered with few L. crispatus-dominant women. Nevertheless, we did observe a nonsignificant trend of reduced HIV incidence with $L$. crispatus-dominant women compared with women who had non-Lactobacillusdominant profiles, regardless of tenofovir or placebo gel assignment.

The ecological diversity in the vaginal microbiome has been previously linked to changes in mucosal immunity in the female genital tract, including elevated cytokine levels, increased HIV target cells (CD4 $4^{+} \mathrm{CCR} 5^{+}$T cells), as well as epithelial barrier disruption $(20,30,31)$. Thus, the presence of BV-associated inflammatory bacteria could have influences at the vaginal mucosa that may add collectively to a multifactorial mechanism affecting microbicide efficacy. The relative contributions of drug depletion and these potential host modulatory effects on microbicide efficacy would be an important avenue of future investigation.

Some limitations need to be taken into account when interpreting these data. The lack of clinical BV measurements, such as Nugent score or Amsel's criteria, precludes our ability to associate these findings with clinical BV criteria, an important factor in vaginal health. Instead, these data are limited to associations with metaproteomic and metagenomic characterization of $\mathrm{BV}$. Another caveat of this study is the assumption 
A

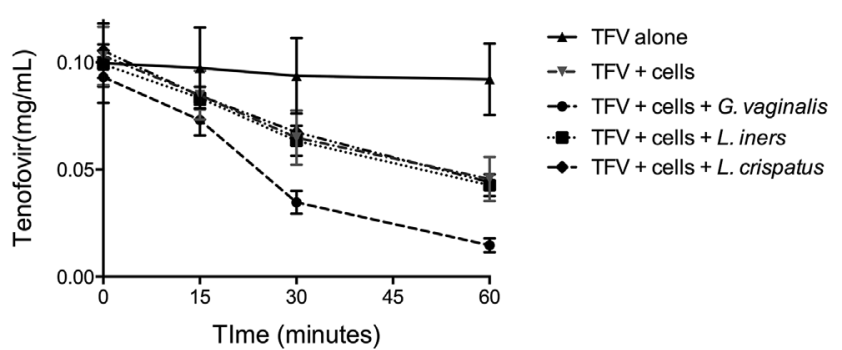

60 minutes:

G. vaginalis vs. TFV + cells: $P=0.0001 \quad$ G. vaginalis vs. $L$. crispatus: $P=0.0238$

G. vaginalis vs. L. iners: $P=0.0022 \quad$ Lactobacillus spp. vs. TFV/cells: $P=n s$

C

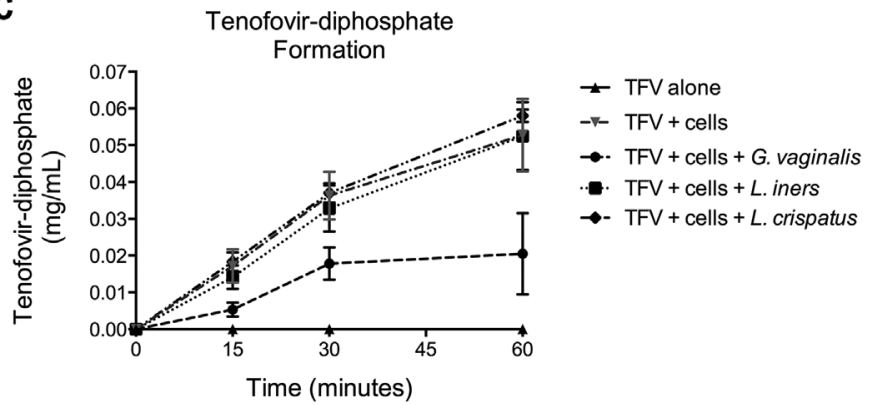

60 minutes:

G. vaginalis vs. TFV + cells: $P=0.0002$

G. vaginalis vs. L. iners: $P=0.0022$

G. vaginalis vs. $L$. crispatus: $P=0.0238$

Lactobacillus spp. vs. TFV/cells: $P=$ ns

Fig. 4. Kinetics of tenofovir depletion by bacteria relative to conversion to pharmacologically active tenofovir diphosphate. In vitro cultures of Jurkat cells ("cells") with tenofovir, cocultured with L. crispatus, L. iners, or G. vaginalis. (A) Tenofovir (TFV) is rapidly decreased in supernatants of cultures including Jurkat cells and most rapidly in cultures with $G$. vaginalis. (B) The metabolite of bacterial metabolism of tenofovir,

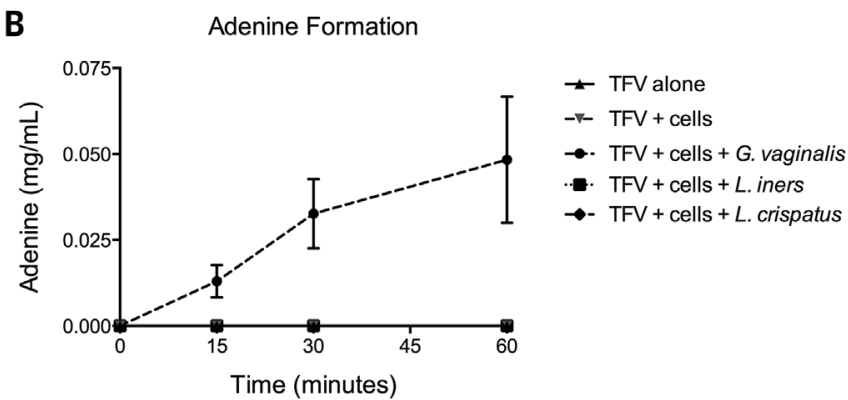

60 minutes:

G. vaginalis vs. TFV + cells: $P=0.0022 \quad$ G. vaginalis vs. $L$. crispatus: $P=0.0022$ G. vaginalis vs. $L$. iners: $P=0.0022$

Lactobacillus spp. vs. TFV/cells: $P=\mathrm{ns}$

D

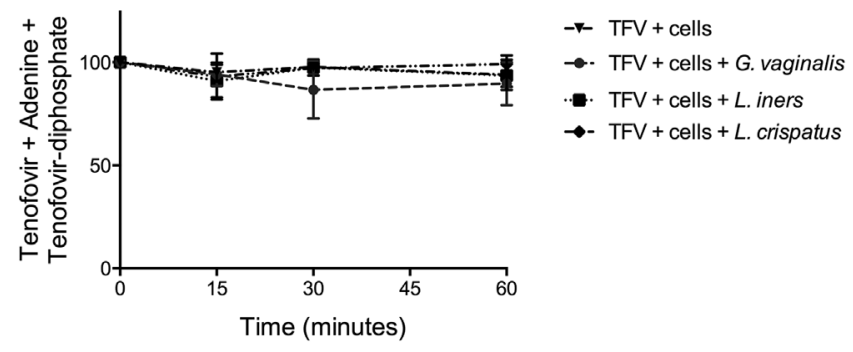

adenine, is only formed in the presence of G. vaginalis. (C) Tenofovir diphosphate (TFV-DP) is produced in Jurkat cells in the presence of $L$. iners and $L$. crispatus but is significantly lower in the presence of $G$. vaginalis. (D) Total drug recovery demonstrates that tenofovir, tenofovir diphosphate, and adenine make up major tenofovir-based drug recovery. Error bars indicate SEM. ns, not significant. that a single random sample represents the vaginal microbiome for the duration of the trial. Previous longitudinal evaluations of women demonstrated that some bacterial communities shift substantially in short periods of time, whereas others, including those dominated by Lactobacillus species, demonstrated considerably more stability, where outgrowth of anaerobic bacteria was less frequent $(\sim 10 \%)$ over time $(32)$. In a sensitivity analysis to address this question, unrealistically high levels of misclassification would be required to double the HIV incidence rate observed in this study. In randomly selected LD women $(n=21), 76 \%$ maintained LD status at previous visits (median days $=301$ ), and a $24 \%$ misclassification had a minimal effect on the overall incidence rate. Further, as neither the trial participants nor investigators were aware of $\mathrm{LD}$ status at randomization, overall random allocation in the CAPRISA 004 trial is expected to lead to comparable groups assigned to study arms of the $\mathrm{LD}$ and non-LD strata.

Although it is a limitation of the data that the metabolism of tenofovir was only demonstrated in vitro here, the decreased tenofovir levels in women with vaginal dysbiosis support this as a potential in vivo mechanism. Further, the in vitro studies were performed with a range from 1 to $10 \mathrm{mg} / \mathrm{ml}$, representing predicted in vivo tenofovir concentrations after gel use (33), and we observed similar kinetics of tenofovir metabolism despite tenofovir concentration. Because multiple BV-associated bacteria could biodegrade tenofovir in vitro, future studies assessing the effects of mixed bacteria cultures on tenofovir metabolism would be an important next step. Though we have not yet assessed whether Pseudomonas species metabolize tenofovir, they (group IIe) represent a small fraction of women (3.5\%), and removal of this group in LD versus non-LD comparisons did not affect the reported outcomes. Other BV-associated bacteria not evaluated in this study may also metabolize tenofovir and would be important to explore. We demonstrated that clinical isolates of $G$. vaginalis are also capable of degrading tenofovir, albeit at reduced rates as compared with type strains, possibly due to decreased growth rates in the clinical isolates. In addition, this mechanism is supported by several studies showing in vivo metabolism of drugs by microbiota, just never in the context of HIV infection and antiretroviral metabolism (34). Furthermore, the kinetics of bacterial metabolism of tenofovir relative to host cellular uptake and conversion of tenofovir to the pharmacologically active phosphorylated form (tenofovir diphosphate) demonstrated that G. vaginalis can actually metabolize tenofovir more rapidly that target cells can convert it to tenofovir diphosphate. Previous studies in CAPRISA 004 demonstrated that low levels of tenofovir diphosphate were associated with infection (35), and a recent study by Hillier et al. demonstrated that, in the FAME study, both genital and plasma levels of tenofovir diphosphate were negatively correlated with G. vaginalis levels in the vagina, as well as markers of BV, demonstrating the clinical relevance of our results (36).

This study used mass spectrometry-based proteomics, an emerging technology for the study of mucosal systems, to characterize vaginal bacteria. Both mass spectrometry and standard $16 S$ rRNA sequencing had high concurrence in the classification of Lactobacillus-dominant profiles. There were some differences in the proportion of detected taxa. Particularly, in mass spectrometry analysis, the predominant genera-such as Lactobacillus, Gardnerella, and Prevotella-more frequently dominate the bacterial proteome load as compared with $16 S$ results, similar to previous observations (20). This could be due to sensitivity differences as the proteome spans a larger dynamic range, posttranscriptional regulatory mechanisms that modulate protein translation from 
genes, a reflection of greater diversity of metabolic states, or divergence of functionality between the same taxa in different individuals or CSTs. Future studies of the bacterial metaproteome may provide further insights into in vivo microbial systems.

We assessed whether LD and non-LD women differed with respect to clinical symptoms associated with STIs and found that reported vaginal symptoms did not differ between $\mathrm{LD}$ and non-LD women. This finding is concordant with other descriptions suggesting that the presence of $G$. vaginalis is often asymptomatic $(11,12)$, making it unlikely that G. vaginalis led to behavioral changes affecting gel use. Whereas previous results showed that tenofovir is protective against HSV-2 (37), our subanalysis did not find an effect of Lactobacillus dominance on efficacy of tenofovir against HSV-2 acquisition. However, given the many differences between HIV and HSV-2, including the target cells in the genital tract and the unknown levels required for protection in vivo, it is challenging to compare these two infections in this context. Future studies aimed at assessing tenofovir in decreased HSV-2 infection relative to vaginal microbiota will be very important.

Because Lactobacillus dominance corresponds with a relatively low vaginal $\mathrm{pH}$, typically below 4.5 (12), vaginal $\mathrm{pH}$ testing may be a pragmatic approach to identify women most likely to benefit from topical tenofovir-containing microbicides and potentially other prevention strategies. However, current treatment strategies for BV may not be sufficiently efficacious (38), and better strategies that can both deplete anaerobic bacteria and support recolonization with Lactobacillus may be required. If validated in other trials, this could be a compelling reason for integrating topical microbicide implementation with sexual and reproductive health services so that vaginal health becomes an integral component of HIV prevention approaches.

\section{Conclusions}

Vaginal microbiota modulated the efficacy of the topical tenofovir microbicide in CAPRISA 004. The rapid depletion of tenofovir by $G$. vaginalis and other BV-associated anaerobic bacteria by metabolism provides a biological mechanism likely contributing to a multifactorial process, including increased vaginal inflammation and adherence, leading to varying levels of HIV prevention efficacy observed across topical microbicide trials. This suggests that women with nonLactobacillus-dominant vaginal bacteria may be more sensitive to the timing of gel application and adherence, whereas bacterial communities with Lactobacillus dominance may facilitate an environment more conducive to topical microbicide efficacy. Thus, vaginal $\mathrm{pH}$ and/or microbiota screening are interventions that can potentially be added to topical microbicide initiation to guide and enhance prevention of HIV in women. These findings provide evidence about the importance of vaginal microbial communities on prevention efficacy, which could help improve this HIVspecific prevention strategy for women.

\section{REFERENCES AND NOTES}

1. Q. Abdool Karim et al., Int. J. Epidemiol. 40, 922-930 (2011).

2. UNAIDS, "Global AIDS update 2016" [Joint United Nations Programme on HIV/AIDS (UNAIDS), 2016].

3. J.-M. Molina et al., N. Engl. J. Med. 373, 2237-2246 (2015).

4. S. McCormack et al., Lancet 387, 53-60 (2016).

5. R. M. Grant et al., N. Engl. J. Med. 363, 2587-2599 (2010).

6. S. S. Abdool Karim, A. D. M. Kashuba, L. Werner, O. Abdool Karim, Lancet 378, 279-281 (2011).

7. M. L. Cottrell et al., J. Infect. Dis. 214, 55-64 (2016).

8. S. Srinivasan et al., PLOS ONE 5, e10197 (2010).

9. S. L. Hillier, N. Engl. J. Med. 353, 1886-1887 (2005).

10. J. H. van de Wijgert et al., PLOS ONE 9, e105998 (2014).

11. D. N. Fredricks, T. L. Fiedler, J. M. Marrazzo, N. Engl. J. Med. 353, 1899-1911 (2005)

12. J. Ravel et al., Proc. Natl. Acad. Sci. U.S.A. 108 (suppl. 1), 4680-4687 (2011)

13. M. N. Anahtar et al., Immunity 42, 965-976 (2015).

14. R. Hummelen et al., PLOS ONE 5, el2078 (2010)

15. R. Amsel et al., Am. J. Med. 74, 14-22 (1983).

16. R. P. Nugent, M. A. Krohn, S. L. Hillier, J. Clin. Microbiol. 29 , 297-301 (1991).

17. H. L. Martin et al., J. Infect. Dis. 180, 1863-1868 (1999).

18. J. Atashili, C. Poole, P. M. Ndumbe, A. A. Adimora, J. S. Smith, AIDS 22, 1493-1501 (2008).

19. L. Masson et al., Clin. Infect. Dis. 61, 260-269 (2015)

20. A. S. Zevin et al., PLOS Pathog. 12, e1005889 (2016).

21. C. Kenyon, R. Colebunders, T. Crucitti, Am. J. Obstet. Gynecol. 209, 505-523 (2013)

22. Q. Abdool Karim et al., Science 329, 1168-1174 (2010).

23. J. M. Marrazzo et al., N. Engl. J. Med. 372, 509-518 (2015)

24. H. Rees et al., paper presented at the Conference on Retroviruses and Opportunistic Infections (CROI), Seattle, WA, 23 to 26 February 2015.

25. S. Pendharkar et al., BMC Infect. Dis. 13, 43 (2013).

26. J. H. van de Wijgert, M. C. Verwijs, A. N. Turner, C. S. Morrison, AIDS 27, 2141-2153 (2013)

27. R. P. Madan et al., PLOS ONE 7, e40415 (2012).
28. H. Borgdorff et al., ISME J. 8, 1781-1793 (2014)

29. C. Gosmann et al., Immunity 46, 29-37 (2017).

30. A. R. Thurman et al., AIDS Res. Hum. Retroviruses 31. 1139-1152 (2015).

31. A. Burgener, I. McGowan, N. R. Klatt, Curr. Opin. Immunol. 36 22-30 (2015)

32. P. Gajer et al., Sci. Transl. Med. 4, 132 ra52 (2012).

33. J. L. Schwartz et al., PLOS ONE 6, e25974 (2011).

34. M. EIRakaiby et al., OMICS 18, 402-414 (2014)

35. A. D. Kashuba et al., J. Acquir. Immune Defic. Syndr. 69, 264-269 (2015)

36. S. L. Hillier et al, paper presented at the Conference on Retroviruses and Opportunistic Infections, Seattle, WA, 13 to 16 February 2017.

37. S. S. Abdool Karim et al., N. Engl. J. Med. 373, 530-539 (2015)

38. K. E. Bunge, R. H. Beigi, L. A. Meyn, S. L. Hillier, Sex. Transm. Dis. 36, 711-713 (2009).

\section{ACKNOWLEDGMENTS}

The data reported in this manuscript are tabulated in the main paper and in the supplementary materials. Funding for this research was provided by the Canadian Institutes of Health Research (CIHR) (A.D.B., L.R.M., S.S.A.K.) (grant TMI 138658), the Department of Pharmaceutics at the University of Washington (N.R.K.), and the Public Health Agency of Canada (A.D.B, G.W.). M.P. is a recipient of a studentship of the CIHR and the Manitoba Health Research Council. We thank S. McCorrister, M. Abou, M. Cook, and C. Miller for technical assistance; N. Yende for statistical support; and J. Schellenberg for primary bacterial isolates. We also thank all investigators of the CIHR Team Grant, including K. Broliden, K. Arnold, D. Lauffenburger, K. Hasselrot, and $A$. Tjernlund. The funding agencies did not have any role in the study design; the collection, analysis, and interpretation of data; or the writing of the paper or its submission for publication. The CAPRISA 004 tenofovir gel trial was funded principally by the U.S. Agency for International Development, grants through $\mathrm{FHI} 360$, and CONRAD for product manufacturing. with support from the South African Department of Science and Technology (DST). We thank the DST and the South African National Research Foundation for supporting the specimen repository; A. Kashuba for tenofovir concentration data; the CAPRISA 004 study team, including J. Frohlich, A. Kharsany, K. Mlisana, C. Baxter, T. Gengiah, N. Samsunder, and S. Sibeko, as well as the study clinicians, counselors, pharmacists, statisticians, and fieldwork, data quality, laboratory and administrative staff for their contributions; and the women who participated in the CAPRISA 004 trial.

\section{SUPPLEMENTARY MATERIALS}

www.sciencemag.org/content/356/6341/938/suppl/DC1

Materials and Methods

Figs. S1 to S9

Tables S1 to S3

References (39-47)

Data S1

16 September 2016; resubmitted 25 January 2017

Accepted 13 April 2017

10.1126/science.aai9383 


\section{Science}

\section{Vaginal bacteria modify HIV tenofovir microbicide efficacy in African women}

Nichole R. Klatt, Ryan Cheu, Kenzie Birse, Alexander S. Zevin, Michelle Perner, Laura Noël-Romas, Anneke Grobler, Garrett Westmacott, Irene Y. Xie, Jennifer Butler, Leila Mansoor, Lyle R. McKinnon, Jo-Ann S. Passmore, Quarraisha Abdool Karim, Salim S. Abdool Karim and Adam D. Burgener

Science 356 (6341), 938-945.

DOI: $10.1126 /$ science.aai9383

\section{Vaginal microbiome influences HIV acquisition}

Tenofovir is a preexposure drug used to prevent HIV infection. In clinical trials, tenofovir was effective for men, but not women. Klatt et al. now show that tenofovir efficacy in women depends on the composition of the vaginal microbiome (see the Perspective by Tuddenham and Ghanem). In a clinical trial of 688 women, tenofovir was three times as effective among those with a Lactobacillus-dominant vaginal microbiome as it was among other women. Gardnerella vaginalis tended to predominate in the women for whom tenofovir was less effective, and the authors found that the organism could rapidly metabolize and thereby inactivate the drug.

Science, this issue p. 938; see also p. 907

ARTICLE TOOLS

SUPPLEMENTARY

MATERIALS

RELATED
CONTENT

REFERENCES

PERMISSIONS http://science.sciencemag.org/content/356/6341/938

http://science.sciencemag.org/content/suppl/2017/05/31/356.6341.938.DC1

http://science.sciencemag.org/content/sci/356/6341/907.full

http://stm.sciencemag.org/content/scitransmed/9/381/eaai7521.full $\mathrm{http}: / /$ stm.sciencemag.org/content/scitransmed/9/377/eaag1809.full http://stm.sciencemag.org/content/scitransmed/9/381/eaai7514.full http://stm.sciencemag.org/content/scitransmed/9/383/eaag0019.full

This article cites 44 articles, 5 of which you can access for free http://science.sciencemag.org/content/356/6341/938\#BIBL

http://www.sciencemag.org/help/reprints-and-permissions

Use of this article is subject to the Terms of Service

Science (print ISSN 0036-8075; online ISSN 1095-9203) is published by the American Association for the Advancement of Science, 1200 New York Avenue NW, Washington, DC 20005. 2017 () The Authors, some rights reserved; exclusive licensee American Association for the Advancement of Science. No claim to original U.S. Government Works. The title Science is a registered trademark of AAAS. 